\title{
Inhibition-related Activation in the Right Inferior Frontal Gyrus in the Absence of Inhibitory Cues
}

\author{
Agatha Lenartowicz ${ }^{1}$, Frederick Verbruggen ${ }^{2}$, Gordon D. Logan ${ }^{3}$, \\ and Russell A. Poldrack ${ }^{4}$
}

\begin{abstract}
The right inferior frontal gyrus (rIFG) has been hypothesized to mediate response inhibition. Typically response inhibition is signaled by an external stop cue, which provides a top-down signal to initiate the process. However, recent behavioral findings suggest that response inhibition can also be triggered automatically by bottom-up processes. In the present study, we evaluated whether rIFG activity would also be observed during automatic inhibition, in which no stop cue was presented and no motor inhibition was actually required. We measured rIFG activation in response to stimuli that were
\end{abstract}

\section{INTRODUCTION}

Inhibition of responses is a core function of cognitive control, which can be broadly defined as the process of deliberately overriding a prepotent or ongoing action. Response inhibition has been extensively studied using the stop signal task and its variants (Verbruggen \& Logan, 2008c; Logan, Cowan, \& Davis, 1984). In this task, participants must respond to stimuli that are presented in a continual stream and must withhold their response upon hearing an infrequent auditory cue. Considerable evidence from neuroimaging research suggests that the right inferior frontal gyrus (rIFG) is implicated in stopping the motor responses. For instance, the BOLD response in this region is elevated during response inhibition relative to routine responding (Aron \& Poldrack, 2006; Rubia, Smith, Brammer, \& Taylor, 2003; Menon, Adleman, White, Glover, \& Reiss, 2001; Garavan, Ross, \& Stein, 1999; Konishi et al., 1999). Moreover, stopping ability is impaired both by damage to this region (Hodgson et al., 2007; Aron, Fletcher, Bullmore, Sahakian, \& Robbins, 2003) and by TMS (Chambers et al., 2006). The question addressed in this study is whether rIFG can be activated by stimuli that were previously associated with stop signals (i.e., automatic inhibition; Verbruggen \& Logan, 2008b) when no stop signals are presented with those stimuli and response inhibition is not required.

\footnotetext{
${ }^{1}$ University of California, Los Angeles, ${ }^{2}$ University of Exeter, ${ }^{3}$ Van-
} derbilt University, Nashville, ${ }^{4}$ University of Texas at Austin previously associated with stop signals but which required a response on the current trial (reversal trials). The results revealed an increase in rIFG (pars triangularis) activity, suggesting that it can be activated by associations between stimuli and stopping. Moreover, its role in inhibition tasks is not contingent on the presence of an external stop cue. We conclude that rIFG involvement in stopping is consistent with a role in reprogramming of action plans, which may comprise inhibition, and its activity can be triggered through automatic, bottom-up processing.

Automatic inhibition was documented by Verbruggen and Logan (2008a, 2008b) to demonstrate that response inhibition need not always occur through a top-down executive pathway, as often inferred (e.g., Ridderinkhof, van den Wildenberg, Segalowitz, \& Carter, 2004; Miyake et al., 2000; Burgess, Alderman, Evans, Emslie, \& Wilson, 1998). Rather, response inhibition can be achieved in one of two ways by a controlled "top-down" process or by an automatic, "bottom-up" process, depending on the consistency of the associations between stop signals and go stimuli. In the automatic inhibition task, associations between particular go stimuli and stop signals are first acquired by systematically pairing the two during training. Then, in a test period, the go stimulus is presented without a stop signal (i.e., as a "go" trial). Verbruggen and Logan (2008a) demonstrated that even when there was no explicit cue to trigger inhibition, the prior association of the stimulus and stop signal was sufficient to slow responses-response inhibition was automatically triggered. If rIFG is critical to both top-down and bottomup response inhibition, we would expect an increase in activity in this region during "go" trials that were previously associated with stopping relative to "go" trials with no prior associations. That is, we would expect automatically triggered inhibition to increase activation within rIFG.

The presence of automatic inhibition effects on rIFG activity would also provide evidence against a sole alternative hypothesis for its function in stopping, based in stimulusdriven attention (Corbetta \& Shulman, 2002). From this perspective rIFG responds to salient cues that are relevant 
to the current task, of which stop signals comprise one exemplar. For instance, the ventrolateral regions of $\mathrm{pFC}$ (including rIFG) are critical to the acquisition and monitoring of stimulus-response mappings in tasks that have no inhibitory component (e.g., working memory; Shallice, Stuss, Alexander, Picton, \& Derkzen, 2008; D’Esposito \& Postle, 2002; Owen, 1997; Stuss, Shallice, Alexander, \& Picton, 1995; Petrides, 1994). Recently, Hampshire, Chamberlain, Monti, Duncan, and Owen (2010) assessed directly whether rIFG activity is more selective for inhibition cues than for other task cues and found no differences. They also suggested that this region is tuned to respond most strongly to any targets that were taskrelevant (Hampshire, Thompson, Duncan, \& Owen, 2009; Hampshire, Duncan, \& Owen, 2007). Hence the role of rIFG during stopping may be to detect the presence of the stop signal, which is a salient and task-relevant stimulus, and subsequently inform the motor system (which implements the inhibition) that the current response should be withheld (e.g., Duann, Ide, Luo, \& Li, 2009).

Thus, if the stimulus-driven attention perspective is correct and rIFG activity reflects a response to the stop signal, then its activity during go trials should be unaffected by prior associations between go stimuli and stop signals (automatic inhibition). Conversely, if rIFG activity is not always contingent on the presence of an external stop signal, then its activity should increase during go trials that were previously paired with stop signals. Such a finding would be evidence that rIFG is involved in response inhibition regardless of whether its implementation occurred through top-down (triggered by the stop signal) or bottom-up (triggered by the association between a go stimulus and the stop signal) processes. These hypotheses were the focus of our study.

\section{METHODS}

\section{Behavioral Methods}

\section{Task Design}

The participants' task was to make gender judgments (male/ female) about face stimuli, and then make a button response to indicate their choice. The stimulus set comprised 50 faces that were selected from an existing set compiled by Neal Cohen of the University of Illinois, Urbana-Champaign. On $24 \%$ of all trials, a tone was presented along with the face stimulus, requiring participants to withhold their response. These were called "stop" trials in contrast to the $76 \%$ of "go" trials during which the response was executed. Participants performed this task in two phases: training and testing. Unbeknown to the participants, the pairings of individual faces and the go or stop responses were varied between these phases such that some faces would be automatically associated with either stopping or going whereas others would not. Then during testing, all faces were systematically paired with either stop or go responses; only the training history differed among face stimuli.
This procedure resulted in the following five types of trials during testing (also shown in Figure 1): go-go, stop-go, go/ stop-go, go-stop, and go/stop-stop. Twelve percent of all stimuli were systematically paired with stopping during training but were reversed to go responses during testing (stop-go). These reversal-go trials should be subject to interference from prior associations with stopping and were of primary interest in the current study. The stop-go trials were compared with the same number of go/stop-go trials. The stimuli for these standard go trials were randomly paired with either stopping or going during training $(p(\mathrm{go})=.5, p($ stop $)=.5)$, but only with going during testing. They, thus, provide a suitable control to stop-go trials.

For comparison, another $12 \%$ of all stimuli were systematically paired with going during training and were switched to stop responses during testing (go-stop). Standard stop trials, like standard go trials, involved stimuli that were randomly paired with stopping or going during training (go/stop-stop). The remaining 52\% of all trials were stimuli that were assigned to go responses across both training and testing (go-go). These trials provided the additional go events that ensured that the ratio of stop to go trials was 24/76 across phases.

A sample trial sequence, which did not differ between phases, is shown in Figure 1. Each trial began with a 500-msec presentation of a white fixation cross, presented centrally on a black background. This was followed by a stimulus presented for 1000 msec. During this time, participants were required to indicate if the face was male or female by pressing one of two buttons as quickly as possible. Response mappings were assigned to middle and index finger of the right hand and to male/female responses, counterbalanced across subjects. The stimulus remained on screen after response for the remainder of the $1000 \mathrm{msec}$. The onset of the subsequent stimulus was delayed by an intertrial interval during which the screen remained blank. This interval duration was varied randomly, according to a continuous exponential distribution with a mean of $1000 \mathrm{msec}$ and range of 500-4000 msec.

On $24 \%$ of all trials, a $900-\mathrm{Hz}$ tone was presented for $500 \mathrm{msec}$, indicating that participants should withhold their response. These stop trials were designed to isolate the duration of the stopping mechanism based on procedures of Logan and Cowan (1984). Namely, the tone was presented with a variable delay relative to face stimulus onset (stop signal delay, SSD) to ensure that the probability of stopping successfully, $p$ (inhibit), converged to .5. To achieve $p$ (inhibit) $=.5$, the SSD was increased by $50 \mathrm{msec}$ if the participants had stopped successfully, thus making stopping more difficult on the next stop trial. Similarly, the SSD was decreased by $50 \mathrm{msec}$ if the participant had failed to stop, thus making the stopping easier on the subsequent stop trial. This staircase procedure ensured that, assuming participants responded as quickly as possible, their performance would converge on $p$ (inhibit) $=.5$. To minimize participants' likelihood of predicting the SSD, two separate staircases were initialized 
Figure 1. Participants made gender judgments in response to face stimuli, pressing a button to indicate their response. On $24 \%$ of all trials, a stop signal (tone) was presented shortly following stimulus onset (SSD). During these trials, participants had to withhold their response. The face stimuli were assigned to one of five train-test configurations. During events of interest (stop-go), faces were systematically paired with stopping during training but required a go response during testing. An effect of automated inhibition would be expected to slow down RTs during these trials and increase activity in rIFG. Note that scanning was performed only during testing.

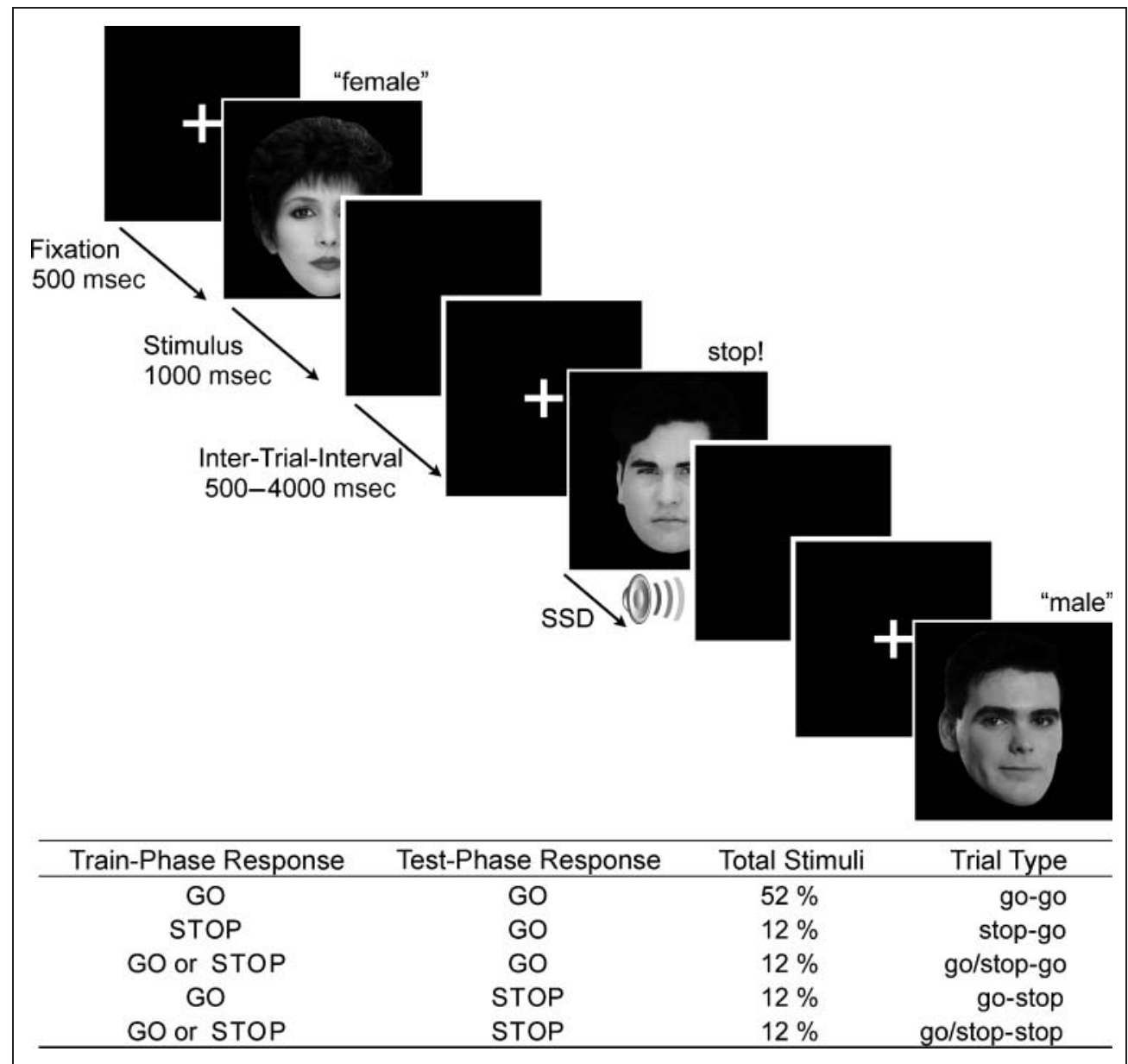

with SSDs of 250 and 350 msec for each of the two unique types of stop trials. The order of the staircases was interleaved across stop trials. The SSD in each staircase for the last 10 trials of each block was used to initialize each of the staircase SSDs during the next block. Finally, an additional tracking procedure was implemented to reduce the tendency to wait for a stop signal (Sylwan, 2004): On each go trial, the RT was compared against the mean RT for all prior go trials in a block. If it exceeded two standard deviations of the mean, a warning signal was presented by changing the color of the subsequent fixation cross to orange. At the completion of each block of trials participants were also presented with feedback, median RT and accuracy for go trials and percentage inhibition for stop trials.

The stop and go trials were presented in blocks of 100 trials. Thus, 12 go-stop trials and 12 go/stop-stop trials were presented in each test block, along with 76 go trials (12 stop-go, 12 go/stop-go, and 52 go-go trials). The stop and go trials, as well as ISIs of varying duration, were distributed pseudorandomly within the block to optimize the efficiency of detecting differences between go and stop trials. Custom Matlab software (MathWorks, Natick, MA) was developed for this purpose based on the methods developed by Liu, Frank, Wong, and Buxton (2001).
We generated one million random event sequences, and for each, we calculated its efficiency for contrasts comparing stop and go trials and for contrasts comparing reversal and nonreversal trials. The efficiency of a contrast was defined as $\left(c^{\mathrm{T}} \times\left(X^{\mathrm{T}} \times X\right)^{-1} \times c\right)^{-1}$ (Dale, 1999), wherein $X$ contains the design matrix (one column for each event regressor, see Model Fitting), $c$ contains the contrast vector, $\mathrm{T}$ is the transpose operator, and -1 indicates the matrix inverse. The top 100 sequences were selected based on the highest efficiency scores combined across all contrasts. The 50 face stimuli were presented twice within each block, ensuring that no stimuli repeated on consecutive trials. Transitions were counterbalanced to ensure comparable sequence effects among trial types. Stimulus presentation was implemented in Matlab, using the Psychophysics Toolbox extensions (Brainard, 1997).

\section{Procedures}

The training and testing phases were distributed across 2 days. During the training, phase participants performed six blocks of the task in a behavioral testing facility. All stimuli were presented on an Apple eMac computer, and tones were presented through noise-attenuating headphones. Responses were performed with the right hand 
using the left and right arrow keys of a standard keyboard. The experimenter was present in the room during the first block to supervise their performance and encourage them to respond as quickly as possible. The second testing session was performed in the scanning facility. Once again, participants were presented with six block of the task. Within the scanner, participants also responded with the right hand on a MRI-compatible button box and stop tones were played through headphones. The first two blocks were an extension of the training phase to refresh participants' memory of the previously learned stimulusresponse associations (Figure 1). The final four blocks of the second session comprised the testing phase; all stimuli were systematically paired with either stopping or going during this phase. Thus, during testing, participants had equal opportunity across trials to learn the new stimulusresponse associations, and only stimulus training history distinguished the trial types.

\section{Behavioral Analysis}

The stop signal RT (SSRT) was obtained by the integration method, which provides an estimate of the duration of the stopping process (Band, van der Molen, \& Logan, 2003; Logan, 1994). SSRT was always calculated in reference to RT for go trials that were not systematically paired to a stimulus during training. Additional measures of interest included the average SSD and $p$ (inhibit) for stop trials, and error rate and median RT for go trials.

The data were analyzed separately for training and testing blocks of the experiment during the second day (scanning session) for both go and stop trials. The training phase analysis was constrained to the first two blocks of the second session, which capture the retained effects of learning from the previous day. A full analysis of the first session is presented in the Appendix. The testing phase analysis was conducted on the last four blocks of the second session. A description of time-on-task effects during this phase is also described in the Appendix.

\section{Imaging Methods}

\section{MRI Data Acquisition}

All MRI data were acquired with a 3-T Siemens (Erlangen, Germany) MAGNETOM Trio MRI scanner. Each block of the test phase constituted a separate scanning run, during which we collected 156 functional T2*-weighted EPIs (slice thickness $=4 \mathrm{~mm}$, 34 slices, repetition time $=2 \mathrm{sec}$; echo time $=30$ msec; flip angle $=90^{\circ}$; matrix $=64 \times 64$; field of view $=192 \mathrm{~mm}$ ). A structural image was also collected using the magnetization-prepared rapid-acquisition gradientecho (MPRAGE) protocol with these parameters: repetition time $=2.53 \mathrm{sec}$, echo time $=3.31 \mathrm{msec}$, field of view $=$ $256 \mathrm{~mm}$, matrix $=256 \times 256$, saggital plane, slice thickness $=1 \mathrm{~mm}$, 160 slices. To facilitate the registration of the functional images to the structural images, we also col- lected a T2-weighted matched-bandwidth high-resolution structural scan with the same slice prescription as the EPIs.

\section{Image Preprocessing and Registration}

All data processing was carried out using FSL software (www.fmrib.ox.ac.uk/fsl; Smith et al., 2004). First, the brain was isolated from the surrounding tissue using the Brain Extraction Tool. Then to correct for subject motion, functional images in each block were realigned to the middle volume by applying a rigid body ( 6 degrees of freedom) transformation using a normalized correlation similarity function with trilinear interpolation. The motion parameters from each transformation were retained for subsequent analysis (see Model Fitting). Data were then spatially smoothed using a 5-mm FWHM Gaussian kernel, temporally filtered using a nonlinear high-pass filter with a 100 -sec cutoff and grand mean intensity normalized. Additionally, the data were denoised by means of probabilistic independent component analysis (Beckmann \& Smith, 2004). Artifact components were labeled within a supervised classification framework (Tohka et al., 2008) and were then subtracted from the data. Last, the functional images were registered to the matched-bandwidth highresolution scan, then to the MPRAGE structural image, and finally into standard [Montreal Neurological Institute (MNI)] space, using affine transformations. The registration from MPRAGE to MNI space was further refined by nonlinear registration (FMRIB's Linear Image Registration Tool).

\section{Model Fitting}

The data were analyzed in FSL by fitting a general linear model to the time series for each voxel. The model contained eight events and six nuisance regressors. Event regressors were constructed for each correct trial type (go-go, stop-go, go/stop-go, go-stop, and go/stop-stop), each failed stop event, and for erroneous go trials. We did not explicitly model trials on which a warning tone occurred because these occurred, on average, in only six trials across the entire testing session $(S E=0.7)$ and with equal relative frequency across trial types. For each regressor, trials were first dummy coded as $1 \mathrm{sec}$, with onset at fixation and duration determined by the offset of the face stimulus and were then convolved with a double-gamma hemodynamic response function (Glover, 1999). Null events (corresponding to blank intertrial intervals) were not modeled and constitute an implicit baseline. Nuisance regressors included the six motion parameters produced by the prior motion correction procedure. Temporal derivatives of each predictor were also included as regressors to increase model sensitivity. The data were prewhitened to correct for temporal autocorrelation (Woolrich, Ripley, Brady, \& Smith, 2001) before the final model fit was estimated using FMRIB's Improved Linear Model. 


\section{Activation Analyses}

Hypotheses were evaluated by comparing parameter magnitudes for regressors of interest within a multilevel linear modeling framework (Beckmann, Jenkinson, \& Smith, 2003), including robust group analysis using outlier inference (Woolrich, 2008). Whole-brain analyses were used to compare activation during standard and reversal trials for both stopping and going. To replicate previous reports of rIFG activation during stopping, two additional contrasts were constructed to compare the parameter estimates for each of the stop trials (go-stop, go/stop-stop) against go trials (go/stop-go). All analyses were evaluated at the group level, using mixed-effects analysis. Group level parameter maps were thresholded using cluster detection statistics, with a height threshold of $z>2.3$ and cluster probability of $p<.05$, and corrected for whole-brain multiple comparisons using Gaussian random field theory (Worsley et al., 1995).

On the basis of a priori hypotheses, an ROI analysis was also performed to evaluate the above effects within rIFG. The ROI for rIFG included the opercularis and triangularis portions of the IFG, which were anatomically defined based on the Harvard-Oxford probabilistic anatomical atlas provided with FSL (see Figure 3). The means of parameter estimates within this ROI were tested at the group level by computing, for each voxel, a threshold-free cluster enhanced $t$ statistic (Smith \& Nichols, 2009). Inferences on these $t$ statistics were performed by means of a permutation test $(n=5040)$, with family wise error controlled at $p<.05$. This inference methodology was selected because, unlike cluster-based approaches, it provides an exact statistic for each voxel within the ROI.

\section{Participants}

All of our participants were recruited from the University of California, Los Angeles, community ( $n=26,15$ women, mean age $=21.3$ years, $S E=0.9)$. Two of these individuals were excluded from final analysis because of poor image quality, defined by the presence of motion that exceeded $3 \mathrm{~mm}$ or variations in intensity that exceeded three times the image average. One additional participant was excluded because of a failure to respond to any stop signals during the scanning session. All participants provided informed consent in accordance with a protocol approved by the University of California, Los Angeles Institutional Review Board.

\section{RESULTS}

\section{Behavioral Results}

Effects of Stop-stimulus Associations on Stopping and Going

The effects of associations between stop signals and go trial stimuli on stopping and going are shown in Figure 2 and Table 1. First, evidence that stop-stimulus associations

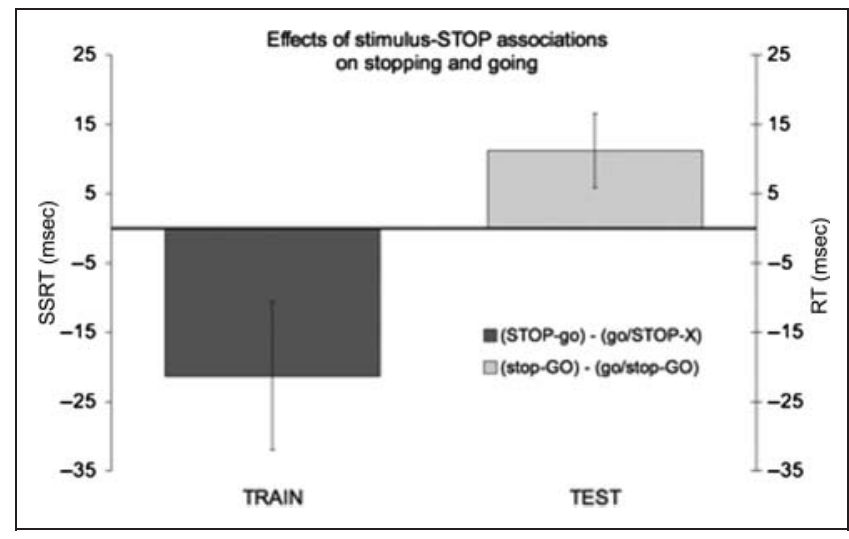

Figure 2. The pairing of stimuli with stopping during training (STOP-go) reduced SSRT as compared with stop trials without such pairings (go/STOP-X), producing a negative difference (left axis and bar). The reversal of the stimulus-stop pairings to stimulus-go pairings during testing (stop-GO), slowed RT during these trials relative to go trials without such learning history (go/stop-GO; right axis and bar). Note that the capitals indicate which trial, within the given training-testing pair, was used in difference calculations.

were acquired during training was revealed by a paired $t$ test; in the training phase of the scan session, mean SSRT was faster during stop trials in which stop signals and go stimuli were paired consistently (216 msec) than during standard stop trials, in which stop signals and go stimuli were not paired systematically (238 $\mathrm{msec}), t(22)=1.99$, $p<.03$ (one-tailed; Figure 2, left). Hence, systematic pairing of go stimuli with stopping had a beneficial effect on SSRT. As expected, $p$ (inhibit) was close to 50\% ( $M=47.8 \%$, $S E=1.5 \%)$ and did not differ among trial types, $t<1$.

The effect of these associations during testing was revealed by slower median RT during stop-go (465 msec) trials than during go/stop-go trials (454 msec), $t(22)=$ $1.94, p<.03$ (one-tailed; Figure 2, right). There was also an effect of trial type on error rate that approached significance, $t(22)=1.93, p<.07$ (two-tailed), reflecting the fact that stop-go trials showed fewer errors (7.4\%) than go/stop-go trials (10.3\%). Thus, stop-stimulus associations had the effect of slowing down RT and increasing accuracy during stop-go trials, consistent with Verbruggen and Logan (2008a).

\section{Effects of Go-stimulus Associations on Going and Stopping}

During training, there was no significant difference between median RT for go trials that were never paired with stop signals (453 $\mathrm{msec}$ ) and go trial stimuli that were inconsistently paired with stop signals (456 msec), $t<1$ (Table 1). Error rate was also comparable (5.3\% vs. $6.7 \%), t(22)=1.05, p<.30$ (two-tailed). These results suggest that go performance failed to benefit from the systematic pairing between stimuli and going during training.

During testing, there was no difference in SSRT between go-stop (220 msec) and go/stop-stop (218 msec) 
Table 1. Summary Behavior Measures for Stop and Go Performance during Scanning

\begin{tabular}{|c|c|c|}
\hline Stimuli Type & Train Phase & Test Phase \\
\hline & \multicolumn{2}{|c|}{ \% Error $(S E)$} \\
\hline go-stop & $0.05(0.02)$ & $0.50(0.01)$ \\
\hline \multirow[t]{2}{*}{ go/stop-stop } & $0.48(0.02)^{\mathrm{a}}$ & $0.50(0.01)$ \\
\hline & \multicolumn{2}{|l|}{$0.07(0.02)^{\mathrm{b}}$} \\
\hline stop-go & $0.52(0.01)$ & $0.07(0.02)$ \\
\hline \multirow[t]{3}{*}{ go/stop-go } & $0.48(0.02)^{\mathrm{a}}$ & $0.10(0.02)$ \\
\hline & \multicolumn{2}{|l|}{$0.07(0.02)^{\mathrm{b}}$} \\
\hline & \multicolumn{2}{|c|}{ SSRT, msec $(S E)$} \\
\hline stop-go & 216 (19) & - \\
\hline go-stop & - & $220(17)$ \\
\hline \multirow[t]{2}{*}{ go/stop-stop } & $238(18)^{a}$ & $218(16)$ \\
\hline & \multicolumn{2}{|c|}{$\mathrm{SSD}, \mathrm{msec}(S E)$} \\
\hline stop-go & $250(24)$ & - \\
\hline go-stop & - & $239(21)$ \\
\hline \multirow[t]{2}{*}{ go/stop-stop } & $232(22)^{a}$ & $242(21)$ \\
\hline & \multicolumn{2}{|c|}{$\mathrm{RT}(S E)$} \\
\hline stop-go & - & $465(13)$ \\
\hline go-stop & $453(11)$ & - \\
\hline go/stop-go & $456(11)^{\mathrm{b}}$ & $454(11)$ \\
\hline
\end{tabular}

Measures contributing to significant effects of stop-stimulus pairings on SSRT during training and RT during testing are indicated in bold. Effects of go-stimulus pairings were not reliable.

${ }^{a}$ Stop trials with randomly paired stimuli during training (regardless of test phase pairing).

${ }^{\mathrm{b}}$ Go trials with randomly paired stimuli during training (regardless of test phase pairing).

trials, $t<1$. The absence of a reversal effect is consistent with the absence of a training effect during go trials. Mean $p$ (inhibit), during this phase, was $50.1 \%(S E=1.0 \%)$. No significant effects were observed for $p$ (inhibit) across trial type, $t<1$, indicating that participants were on task.

\section{Imaging Results}

Because of the a priori hypothesis of rIFG activity for this contrast of stop-go trials versus go/stop-go trials, a selective analysis was performed correcting only for voxels within the independently defined rIFG ROI. This critical analysis revealed a cluster of 34 voxels in rIFG (pars triangularis, $x=52, y=22, z=-6$ ) that was significantly more active during stop-go trials than during go/stop-go trials (Figure 3). This finding is consistent with the RT difference in the test phase and indicates that rIFG activity was not contingent on the presence of external task cues or on the actual inhibition of a motor response. The same contrast was also examined using whole-brain analysis to test for involvement of other regions; no significant regions were present. For the opposite contrast (go/stop-go > stop-go), three regions showed significant activation (Figure 4): left parietal operculum cortex $(x=-34, y=-40, z=28$; cluster size $=1071$ voxels $)$, right superior parietal lobule $(x=$ $20, y=-36, z=66$; cluster size $=1071$ voxels), and left lateral occipital cortex $(x=-46, y=-72, z=6$; cluster size $=985$ voxels).

Analyses comparing go-stop and go/stop-stop trials showed no significant differences in activity at whole-brain corrected levels or within the rIFG ROI analysis. This null result is consistent with a lack of learning or reversal effects in SSRT.

The results of the go/stop-stop versus go/stop-go comparisons (i.e., comparison of stop and go processes) are shown in Figure 5 and Table 2. Consistent with previous reports of stop $>$ go activity, rIFG was more active in go/stop-stop trials than in go/stop-go trials. For the comparison of the reversal go-stop trials versus go/stop-go trials, only activity in left planum temporale and right supramarginal gyrus was significant at a corrected level. rIFG did not show a significant effect for the go-stop $>$ go/ stop-go contrast neither in the whole-brain analysis nor within the rIFG ROI ( $p>.14$, corrected). We performed a conjunction analysis (Nichols, Brett, Andersson, Wager, \& Poline, 2005) to identify regions that were significant in both contrasts; two regions met the criterion, one in right middle ( $x=64, y=-36, z=0)$ and one in superior temporal gyrus ( $x=68, y=-30, z=12$ ), but none in rIFG (see Figure 5). This suggests that rIFG involvement in stopping was not reliable in the go-stop trials, although participants were able to successfully inhibit their responses; however, it did not differ significantly from activity on go/ stop-stop trials.

Reduced activity during go/stop-stop trials compared with go/stop-go trials was observed in right precentral gyrus and hippocampus, as well as left frontal pole, temporal fusiform gyrus and precuneus. No regions were significantly less active during go-stop trials compared with go/stop-go trials.

\section{DISCUSSION}

The objective of our study was to test whether rIFG could be activated automatically during test trials in which no stop signal was presented. We found that cortex within pars triangularis of rIFG showed increased activation during go trials in which stimuli had prior associations with stopping. There was no external stop signal during go trials suggesting that rIFG activity was not contingent on external cues, and its function is, therefore, not limited to stimulus-driven attention.

The increase in rIFG activity during stop-go trials is evidence that rIFG is responsive to learned stimulus-stop associations. Its activation must, therefore, represent either 
Figure 3. The ROI analysis (cf. Imaging Results) revealed a small cluster of voxels within rIFG (pars triangularis, $x=$ $52, y=22, z=-6$ ) in which the BOLD response increased during stop-go trials relative to go/stop-go trials $(p<$ .05 , corrected for multiple comparisons). Gray shading indicates mask region. Significant activation effects are marked in red.

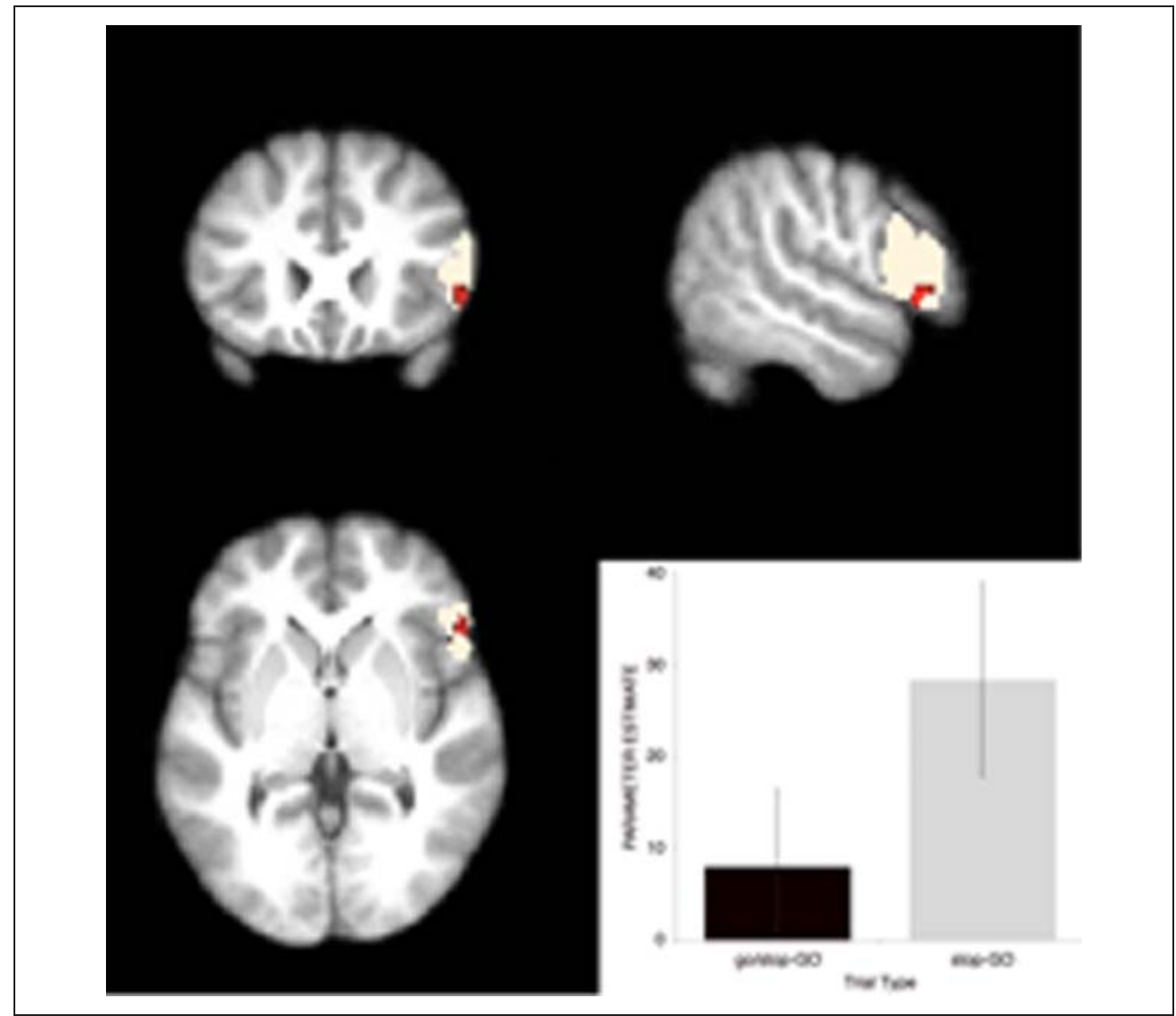

these associations or an inhibitory signal, triggered by stimulus-stop associations that are stored elsewhere. In either case, its function would be consistent with triggering motor inhibition (Aron, 2007). The distinction between these two alternatives is interesting because it highlights a relevant and currently debated issue. Namely, if stimulusstop associations trigger rIFG, then it may be specialized for inhibiting responses. In contrast, if rIFG encodes stimulus-stop associations, then it may not be dedicated to inhibition; it may encode any behaviorally important or systematically occurring association between stimuli and responses. This point was recently emphasized by Hampshire and colleagues, who showed that rIFG responses to target stimuli increase with task relevance (Hampshire et al., 2009) and increase as much with cues that require a response as with cues that indicate response inhibition (Hampshire et al., 2010). This interpretation is also consistent with the finding that IFG activity correlates with acquisition of arbitrary stimulus-response associations (Passingham \& Toni, 2001; Wallis, Dias, Robbins, \& Roberts, 2001), and rIFG damage impairs learning regardless of inhibition (Passingham, Toni, \& Rushworth, 2000; Petrides, 1997; Petrides \& Milner, 1982). The current experiment does not distinguish between these alternative

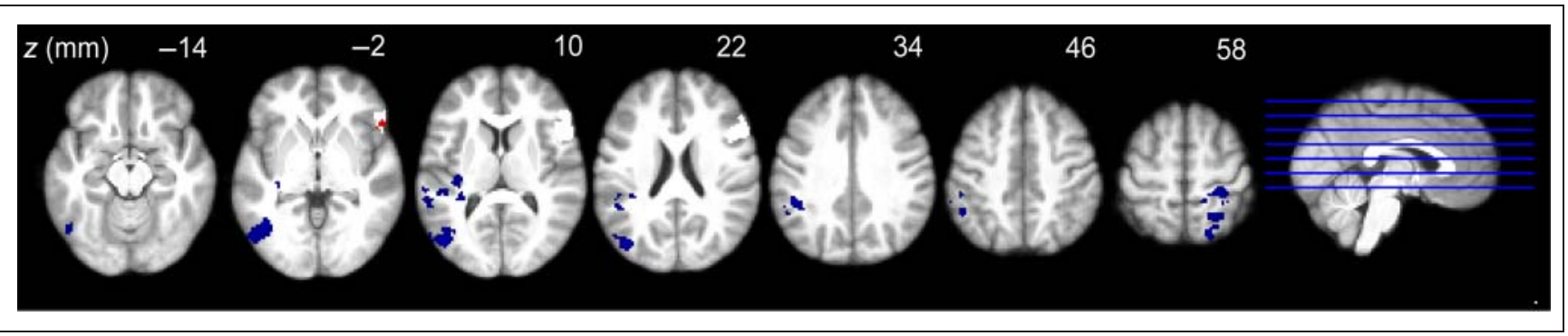

Figure 4. In the whole-brain analysis, three regions were significantly less active ( $p<.05, z>2.3$, corrected for multiple comparisons) during stop-go trials than during go/stop-go trials: left parietal operculum cortex $(x=-34, y=-40, z=28)$, right superior parietal lobule ( $x=20$, $y=-36, z=66)$, and left lateral occipital cortex $(x=-46, y=-72, z=6)$. The reverse contrast showed no significant effects. No differences were observed in the corresponding analysis of go/stop-stop and go-stop trials. As in Figure 3, gray shading indicates extent of rIFG ROI. 


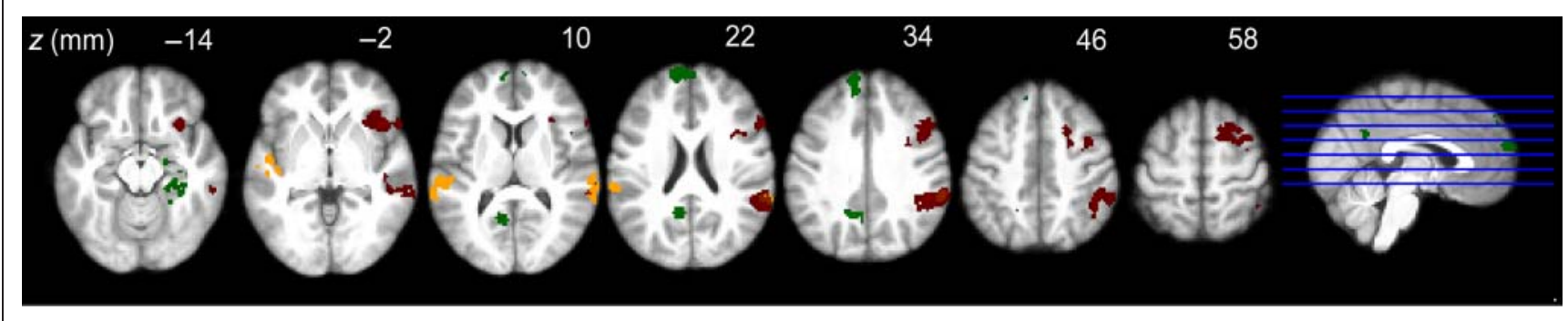

Figure 5. Regions that were significantly ( $p<.05, z>2.3$, corrected for multiple comparisons) more active during stop trials than go trials are shown in red or yellow. Regions that were less active during stop trials than during go trials are shown in green. The rIFG was present only in the go/stop-stop $>$ go/stop-go contrast (red) but not in the go-stop $>$ go/stop-go contrast (orange). The right supramarginal gyrus (see overlap of red and orange at slice at $z=22$ ) was the only region that passed threshold across both contrasts.

roles. Another plausible explanation is that rIFG activity during stop-go trials reflects surprise. This explanation, however, seems unlikely given that this region did not increase in activity during go-stop trials, which would also elicit surprise.

An emerging view is that rIFG is heterogeneous in function, with different subregions supporting different stages of rule processing, namely, detection of change versus updating of the response rules in working memory. Chikazoe et al. $(2008,2009)$ dissociated activity in the more dorsal inferior frontal junction (IFJ; $x=48, y=4, z=38$ ), which increased for infrequent go trials more than for frequent go trials, from activity in the more ventral rIFG $(x=56$, $y=16, z=18$ ), which increased for infrequent stop trials more than for infrequent go trials. The authors concluded that the more dorsal IFJ is involved in detection of change in the environment, the more ventral rIFG is involved in response inhibition. Verbruggen, Aron, Stevens, and Chambers (2010) have recently validated this distinction between IFJ and rIFG by using TMS to disrupt activity in each region during response selection and inhibition. Disruption of IFJ activity (mean stimulation site across subjects: $x=56, y=16, z=33$ ) produced RT slowing consistent with failures in initial stimulus processing (e.g., visual). In contrast, disruption of rIFG (mean stimulation site across subject: $x=58, y=18, z=4$ ) produced RT slowing consistent with action updating. These observations are consistent with the first interpretation above, that rIFG encoded the relevant stimulus-stop associations in the current study. Indeed the activations that we reported fell within the pars triangularis region of $\operatorname{rIFG}(x=52, y=$ $22, z=-6)$. This region is proximate to the coordinates reported by Hampshire et al. (2010) during cue processing $(x=42, y=18, z=-6)$, as well as those reported in the preceding studies (Verbruggen et al., 2010; Chikazoe et al., 2008, 2009) for conditions during which response rules were updated. Interestingly, no IFJ activity was observed during stop-go trials relative to go/stop-go trials, which is consistent

Table 2. Cluster and Peak Voxel Characteristics for Regions that Showed Significant Differences between Stop and Go Trials in Whole-brain Analysis

\begin{tabular}{|c|c|c|c|c|c|c|}
\hline Contrast & \# Voxels & $\operatorname{Max} Z$ & Region & $x$ & $y$ & $z$ \\
\hline \multirow[t]{7}{*}{ go/stop-stop > go/stop-go } & \multirow[t]{6}{*}{1861} & 3.69 & $\mathrm{R}$ inferior frontal gyrus & 32 & 20 & -12 \\
\hline & & 2.50 & $\mathrm{R}$ frontal operculum & 44 & 20 & -2 \\
\hline & & 3.45 & $\mathrm{R}$ inferior frontal gyrus & 58 & 20 & 0 \\
\hline & & 3.44 & paracingulate gyrus & 6 & 20 & 44 \\
\hline & & 3.39 & $\mathrm{R}$ insular cortex & 34 & 18 & -2 \\
\hline & & 3.33 & $\mathrm{R}$ middle frontal gyrus & 42 & 6 & 48 \\
\hline & 1707 & 3.80 & R supramarginal gyrus & 50 & -42 & 32 \\
\hline \multirow[t]{3}{*}{ go/stop-stop $<$ go/stop-go } & 601 & 3.27 & L superior frontal gyrus & -4 & 66 & 20 \\
\hline & 472 & 3.58 & $\mathrm{~L}$ precuneus & -12 & -56 & 32 \\
\hline & 451 & 3.59 & $\mathrm{R}$ hippocampus & 24 & -14 & -22 \\
\hline \multirow[t]{2}{*}{ go-stop > go/stop-go } & 528 & 3.52 & R supramarginal gyrus & 66 & -36 & 16 \\
\hline & 472 & 3.60 & L planum temporale & -54 & -18 & 4 \\
\hline
\end{tabular}

Peak locations are in the coordinate system of MNI ( $\mathrm{mm})$. 
with the absence of explicit cues to indicate change during the former. The increase of rIFG (pars triangularis) activity during stop-go trials in the current study may, therefore, be interpreted as involuntary (and inappropriate) updating of responses according to previously learned associations between stimuli and stopping; although the specific computation performed in this region (e.g., reprogramming of stimulus-response mappings) remains to be determined. Finally, given the subtlety of the activation results, we do not discount that other parts of the rIFG may show similar effects given more powerful experimental manipulation.

An important conclusion from these observations is that control of behavior, which includes response inhibition, involves both top-down ("controlled") and bottom-up ("automatic") processes. In most studies of response inhibition, the control processes is triggered via the top-down pathway, by an external stop cue (reviewed by Verbruggen \& Logan, 2008c; Aron, 2007). However, when stopping is systematically paired with a stimulus, that stimulus can subsequently act as a bottom-up trigger for the inhibitory process (Verbruggen \& Logan, 2008a, 2008b). Accordingly, associations between stimuli and stopping may not require awareness to impact brain activity and behavior. Consistent with this prediction van Gaal, Ridderinkhof, Scholte, and Lamme (2010) reported that unconsciously presented no-go stimuli increased activity in rIFG relative to unconsciously presented go stimuli. Moreover, the degree of this increase correlated positively with RT slowing. ${ }^{1}$ Automatic triggering of this sort has also been recently described for noninhibitory control processes such as conflict monitoring (van Gaal, Lamme, \& Ridderinkhof, 2010), error processing (Cohen, van Gaal, Ridderinkhof, \& Lamme, 2009), and task interference (Lau \& Passingham, 2007), suggesting that bottom-up and top-down interactions are a core component of behavior control.

\section{Conclusion}

In the current study, we observed that activity in rIFG can be elicited by stimuli that were previously associated with stopping, confirming the hypothesis that inhibition in rIFG may be activated automatically and ruling out the hypothesis that the role, at least in the pars triangularis, of rIFG in inhibition is dependent on the externally presented stop cue. Our findings are consistent with recent proposals that rIFG is critical in updating or reprogramming of internally represented motor plans, which may include response inhibition.

\section{APPENDIX}

\section{Training Phase Effects}

Training effects during the first session were evaluated by a repeated measures ANOVA with factors including Time and Trial Type. Time was defined by dividing the session, a total of six blocks, into thirds and averaging across consecutive pairs of blocks. Trial type included reversal (consistent stop-stimulus or go-stimulus pairings) and nonreversal trials (random stop-stimulus or go-stimulus pairings). An additional a priori paired $t$ test was conducted on performance measures from the last third of training, because the effect of systematic stimulus-behavior pairings should be greatest near the end of the session. We expected faster SSRT and RT for systematically paired trials (stimulus-stop and stimulus-go) than randomly paired trials (stimulus-go or stimulus-stop).

\section{Stop Trials}

During training, there were no significant main effects or interactions in SSRT, $F<1.71, p>.19$, or in error rate, $F<2.3, p>.13$, during stop trials. The mean SSRT was $222 \mathrm{msec}$, and error rate was 48.3\% (Table A1). The a priori paired test revealed a trend learning effect on SSRT during the final third of the session, $t(22)=1.2, p<.12$ (onetailed). SSRT was faster during stop-go trials (218 msec) than during randomly matched stimulus-stop trials (229 msec; go/stop-go or go/stop-stop). The same test on error rate was not significant, $t<1$.

\section{Go Trials}

During training, the main effect of Time on RT was significant, $F(2,44)=19.8, p<.001$, indicating an overall decrease in RT across session thirds (503, 468, and $457 \mathrm{msec})$. The main effect of Trial Type was not significant. However, the interaction was statistically reliable, $F(2,44)=3.5, p<.05$. This interaction occurred because for go-stop trials RT decreased linearly across the session, whereas for go/ stop-go and go/stop-stop trials RT decreased most strongly in the first two thirds of the session (Table A2). In error rates, only the main effect of Time was significant, $F(2$, $44)=4.0, p<.03$, because of error rate increasing across session $(4.8 \%, 7.1 \%, 9.2 \%)$.

The a priori paired $t$ test on the final third of the session showed a trend effect of learning on RT, $t(22)=1.47$,

Table A1. Stop Trial Performance during Training (Visit 1)

\begin{tabular}{lccc}
\hline & Blocks 1-2 & Blocks 3-4 & Blocks 5-6 \\
\hline stop-go & $229(11)$ & $215(12)$ & $218(20)$ \\
go/stop-X & $219(12)$ & $227(17)$ & $229(22)$ \\
difference & $10(9)$ & $-12(11)$ & $-11(9) *$ \\
& & \% Error (SE) & \\
stop-go & $0.48(0.02)$ & $0.47(0.02)$ & $0.51(0.02)$ \\
go/stop-X & $0.49(0.02)$ & $0.45(0.02)$ & $0.50(0.02)$ \\
difference & $-0.01(0.01)$ & $0.02(0.02)$ & $0.01(0.02)$ \\
\hline * $p<.12$ (one-tailed). &
\end{tabular}


Table A2. Go Trial Performance during Training (Visit 1)

\begin{tabular}{lccc}
\hline & Blocks 1-2 & Blocks 3-4 & Blocks 5-6 \\
\hline go-STOP & $503(13)$ & $474(11)$ & $451(11)$ \\
go/stop-X & $502(13)$ & $461(8)$ & $462(10)$ \\
difference & $1(6)$ & $13(5)$ & $-11(8)^{*}$ \\
go-STOP & $0.04(0.01)$ & $0.07(0.01)$ & $0.09(0.02)$ \\
go/stop-X & $0.05(0.01)$ & $0.08(0.01)$ & $0.09(0.02)$ \\
difference & $-0.01(0.02)$ & $0.01(0.01)$ & $0.00(0.02)$ \\
\hline
\end{tabular}

$* p<.07$ (one-tailed).

$p<.07$ (one-tailed), because systematically paired go-stop trials had faster RT (451 msec) than randomly paired go/ stop-go go/stop-stop trials $(462 \mathrm{msec})$. This effect was not significant in error rate.

\section{Testing Phase: Time-on-Task Effects}

Time-on-task effects during the testing phase were evaluated by a repeated measures ANOVA with factors including Time and Trial Type. Time was defined by dividing the session, a total of four blocks, into halves and averaging across consecutive pairs of blocks. Separate analyses were conducted for go (RT and error rates) and stop trials (SSRT), with Trial Type (reversal versus random stimulus-response pairing during training) and Time (blocks 1-2 vs. blocks 34) as independent variables. The same analyses were also conducted on fMRI data; however, no significant effects were detected in these analyses. As such, only behavioral effects are reported.

\section{Stop Trials}

There was a significant effect of Block on SSRT, $F(1,23)=$ $15.1, p<.01$, indicating that SSRTs were faster in the second half of the session (203 msec) than in the first half of the session $(231 \mathrm{msec})$. The interaction between Trial Type and Block was not significant, $F(1,23)<1$.

\section{Go Trials}

There was a significant effect of Block on RT, $F(1,23)=$ $15.1, p<.01$, indicating that RTs were faster in the second half of the session $(449 \mathrm{msec})$ than in the first half of the session (463 msec). The interaction between Block and Trial Type was not significant, $F(1,23)<1.9$. There was also a significant effect of Block on error rates, $F(1,23)=5.2$, $p<.03$, indicating that error rates were greater in the second half of the session (10.8\%) than in the first half (7.0\%). In the error rate analysis, the interaction between Trial Type and Block approached significance, $F(1,23)=3.7$, $p<.07$, reflecting the fact that the effect of trial type on error rates was greater in the first half of the session (go/ stop-go: $9.2 \%$, stop-go: $4.7 \%$ ) than in the second half of the session (go/stop-go: 10.9\%, stop-go: 10.7\%).

\section{Acknowledgments}

This work was supported by a 21st Century Science Award from the James S. McDonnell Foundation to R. A. P. and by grant BCS 0957074 from National Science Foundation awarded to G. D. L. F. V. was supported by the Research Foundation Flanders.

Reprint requests should be sent to Agatha Lenartowicz, UCLA Semel Institute for Neuroscience and Human Behavior, 760 Westwood Plaza, Suite 17-369, Los Angeles, CA 90095, or via e-mail: alenarto@ ucla.edu.

\section{Note}

1. We do not report correlations between BOLD signal and (SS)RT in the current study because of our sample size $(n=$ 23), although sufficient to detect activation effects, does not provide sufficient power to reliably identify brain-behavior correlations (cf. Yarkoni, 2009).

\section{REFERENCES}

Aron, A. R. (2007). The neural basis of inhibition in cognitive control. Neuroscientist, 13, 214-228.

Aron, A. R., Fletcher, P. C., Bullmore, E. T., Sahakian, B. J., \& Robbins, T. W. (2003). Stop signal inhibition disrupted by damage to right inferior frontal gyrus in humans. Nature Neuroscience, 6, 115-116.

Aron, A. R., \& Poldrack, R. A. (2006). Cortical and subcortical contributions to stop signal response inhibition: Role of the subthalamic nucleus. Journal of Neuroscience, 26, 2424-2433.

Band, G. P. H., van der Molen, M. W., \& Logan, G. D. (2003). Horse-race model simulations of the stop signal procedure. Acta Psychologica, 112, 105-142.

Beckmann, C. F., Jenkinson, M., \& Smith, S. M. (2003). General multilevel linear modeling for group analysis in fMRI. Neuroimage, 20, 1052-1063.

Beckmann, C. F., \& Smith, S. A. (2004). Probabilistic independent component analysis for functional magnetic resonance imaging. IEEE Transactions on Medical Imaging, 23, 137-152.

Brainard, D. H. (1997). The psychophysics toolbox. Spatial Vision, 10, 433-436.

Burgess, P. W., Alderman, N., Evans, J., Emslie, H., \& Wilson, B. A. (1998). The ecological validity of tests of executive function. Journal of the International Neuropsychological Society, 4, 547-558.

Chambers, C. D., Bellgrove, M. A., Stokes, M. G., Henderson, T. R., Garavan, H., Robertson, I. H., et al. (2006). Executive "brake failure" following deactivation of human frontal lobe. Journal of Cognitive Neuroscience, 18, 444-455.

Chikazoe, J., Jimura, K., Asari, T., Yamashita, K., Morimoto, H., Hirose, S., et al. (2008). Different cognitive control processes implemented by two subregions in inferior frontal cortex during go/no-go task. Neuroscience Research, 61, S65.

Chikazoe, J., Jimura, K., Asari, T., Yamashita, K., Morimoto, H., Hirose, S., et al. (2009). Functional dissociation in right inferior frontal cortex during performance of go/no-go task. Cerebral Cortex, 19, 146-152. 
Cohen, M. X., van Gaal, S., Ridderinkhof, K. R., \& Lamme, V. A. F. (2009). Unconscious errors enhance prefrontal-occipital oscillatory synchrony. Frontiers in Human Neuroscience, 3, 54 .

Corbetta, M., \& Shulman, G. L. (2002). Control of goal-directed and stimulus-driven attention in the brain. Nature Reviews Neuroscience, 3, 201-215.

Dale, A. M. (1999). Optimal experimental design for event-related fMRI. Human Brain Mapping, 8, 109-114.

D'Esposito, M., \& Postle, B. R. (2002). The organization of working memory function in lateral prefrontal cortex: Evidence from event-related functional MRI. In R. T. Knight \& D. T. Stuss (Eds.), Principles of frontal lobe function (pp. 168-187). London: Oxford University Press.

Duann, J. R., Ide, J. S., Luo, X., \& Li, C. R. (2009). Functional connectivity delineates distinct roles of the inferior frontal cortex and presupplementary motor area in stop signal inhibition. Journal of Neuroscience, 29, 10171-10179.

Garavan, H., Ross, T. J., \& Stein, E. A. (1999). Right hemispheric dominance of inhibitory control: An event-related functional MRI study. Proceedings of the National Academy of Sciences, U.S.A., 96, 8301-8306.

Glover, G. H. (1999). Deconvolution of impulse response in event-related BOLD fMRI. Neuroimage, 9, 416-429.

Hampshire, A., Chamberlain, S. R., Monti, M. M., Duncan, J., \& Owen, A. M. (2010). The role of the right inferior frontal gyrus: Inhibition and attentional control. Neuroimage, 50, 1313-1319.

Hampshire, A., Duncan, J., \& Owen, A. M. (2007). Selective tuning of the blood oxygenation level-dependent response during simple target detection dissociates human frontoparietal subregions. Journal of Neuroscience, 27, 6219-6223.

Hampshire, A., Thompson, R., Duncan, J., \& Owen, A. M. (2009). Selective tuning of the right inferior frontal gyrus during target detection. Cognitive Affective \& Behavioral Neuroscience, 9, 103-112.

Hodgson, T., Chamberlain, M., Parris, B., James, M., Gutowski, N., Husain, M., et al. (2007). The role of the ventrolateral frontal cortex in inhibitory oculomotor control. Brain, 130, 1525-1537.

Konishi, S., Nakajima, K., Uchida, I., Kikyo, H., Kameyama, M., \& Miyashita, Y. (1999). Common inhibitory mechanism in human inferior prefrontal cortex revealed by event-related functional MRI. Brain, 122, 981-991.

Lau, H. C., \& Passingham, R. E. (2007). Unconscious activation of the cognitive control system in the human prefrontal cortex. Journal of Neuroscience, 27, 5805-5811.

Liu, T. T., Frank, L. R., Wong, E. C., \& Buxton, R. B. (2001). Detection power, estimation efficiency, and predictability in event-related fMRI. Neuroimage, 13, 759-773.

Logan, G. D. (1994). On the ability to inhibit thought and action: A users' guide to the stop signal paradigm. In D. Dagenbach \& T. H. Carr (Eds.), Inbibitory processes in attention, memory and language (pp. 189-239). San Diego, CA: Academic Press.

Logan, G. D., \& Cowan, W. B. (1984). On the ability to inhibit thought and action-A theory of an act of control. Psychological Review, 91, 295-327.

Logan, G. D., Cowan, W. B., \& Davis, K. A. (1984). On the ability to inhibit simple and choice reaction time responses: A model and a method. Journal of Experimental Psychology: Human Perception and Performance, 10, 276-291.

Menon, V., Adleman, N. E., White, C. D., Glover, G. H., \& Reiss, A. L. (2001). Error-related brain activation during a go/no-go response inhibition task. Human Brain Mapping, 12, 131-143.

Miyake, A., Friedman, N. P., Emerson, M. J., Witzki, A. H., Howerter, A., \& Wager, T. D. (2000). The unity and diversity of executive functions and their contributions to complex "frontal lobe" tasks: A latent variable analysis. Cognitive Psychology, 41, 49-100.

Nichols, T., Brett, M., Andersson, J., Wager, T., \& Poline, J. B. (2005). Valid conjunction inference with the minimum statistic. Neuroimage, 25, 653-660.

Owen, A. M. (1997). The functional organization of working memory processes within human lateral cortex: The contribution of functional imaging. European Journal of Neuroscience, 9, 1329-1339.

Passingham, R. E., \& Toni, I. (2001). Contrasting the dorsal and ventral visual systems: Guidance of movement versus decision making. Neuroimage, 14, S125-S131.

Passingham, R. E., Toni, I., \& Rushworth, M. F. S. (2000). Specialisation within the prefrontal cortex: The ventral prefrontal cortex and associative learning. Experimental Brain Research, 133, 103-113.

Petrides, M. (1994). Frontal lobes and working memory: Evidence from investigations of the effects of cortical excisions in nonhuman primates. In F. Boller \& J. Grafman (Eds.), Handbook of neuropsychology (Vol. 9, pp. 59-82). Amsterdam: Elsevier.

Petrides, M. (1997). Visuo-motor conditional associative learning after frontal and temporal lesions in the human brain. Neuropsychologia, 35, 989-997.

Petrides, M., \& Milner, B. (1982). Deficits on subject-ordered tasks after frontal- and temporal-lobe lesions in man. Neuropsychologia, 20, 249-262.

Ridderinkhof, K. R., van den Wildenberg, W. P. M., Segalowitz, S. J., \& Carter, C. S. (2004). Neurocognitive mechanisms of cognitive control: The role of prefrontal cortex in action selection, response inhibition, performance monitoring, and reward-based learning. Brain and Cognition, 56, 129-140.

Rubia, K., Smith, A. B., Brammer, M. J., \& Taylor, E. (2003). Right inferior prefrontal cortex mediates response inhibition while mesial prefrontal cortex is responsible for error detection. Neuroimage, 20, 351-358.

Shallice, T., Stuss, D. T., Alexander, M. P., Picton, T. W., \& Derkzen, D. (2008). The multiple dimensions of sustained attention. Cortex, 44, 794-805.

Smith, S. M., Jenkinson, M., Woolrich, M. W., Beckmann, C. F., Behrens, T. E. J., Johansen-Berg, H., et al. (2004). Advances in functional and structural MR image analysis and implementation as FSL. Neuroimage, 23, S208-S219.

Smith, S. M., \& Nichols, T. E. (2009). Threshold-free cluster enhancement: Addressing problems of smoothing, threshold dependence and localisation in cluster inference. Neuroimage, 44, 83-98.

Stuss, D. T., Shallice, T., Alexander, M. P., \& Picton, T. W. (1995). A multidisciplinary approach to anterior attentional functions. Structure and Functions of the Human Prefrontal Cortex, 769, 191-211.

Sylwan, R. P. (2004). The control of deliberate waiting strategies in a stop signal task. Brazilian Journal of Medical and Biological Research, 37, 853-862.

Tohka, J., Foerde, K., Aron, A. R., Tom, S. M., Toga, A. W., \& Poldrack, R. A. (2008). Automatic independent component labeling for artifact removal in fMRI. Neuroimage, 39, $1227-1245$.

van Gaal, S., Lamme, V. A. F., \& Ridderinkhof, K. R. (2010). Unconsciously triggered conflict adaptation. Plos One, 5, e11508.

van Gaal, S., Ridderinkhof, K. R., Scholte, H. S., \& Lamme, V. A. F. (2010). Unconscious activation of the prefrontal no-go network. Journal of Neuroscience, 30, 4143-4150.

Verbruggen, F., Aron, A. R., Stevens, M. A., \& Chambers, C. D. (2010). Theta burst stimulation dissociates attention 
and action updating in human inferior frontal cortex. Proceedings of the National Academy of Sciences, U.S.A., 107, 13966-13971.

Verbruggen, F., \& Logan, G. D. (2008a). Automatic and controlled response inhibition: Associative learning in the go/no-go and stop signal paradigms. Journal of Experimental Psychology: General, 137, 649-672.

Verbruggen, F., \& Logan, G. D. (2008b). Long-term aftereffects of response inhibition: Memory retrieval, task goals, and cognitive control. Journal of Experimental Psychology: Human Perception and Performance, 34, 1229-1235.

Verbruggen, F., \& Logan, G. D. (2008c). Response inhibition in the stop signal paradigm. Trends in Cognitive Sciences, $12,418-424$.

Wallis, J. D., Dias, R., Robbins, T. W., \& Roberts, A. C. (2001). Dissociable contributions of the orbitofrontal and lateral prefrontal cortex of the marmoset to performance on a detour reaching task. European Journal of Neuroscience, 13, 1797-1808.

Woolrich, M. (2008). Robust group analysis using outlier inference. Neuroimage, 41, 286-301.

Woolrich, M., Ripley, B. D., Brady, M., \& Smith, S. M. (2001). Temporal autocorrelation in univariate linear modeling of fMRI data. Neuroimage, 14, 1370-1386.

Worsley, K. J., Marrett, S., Neelin, P., Vandal, A. C., Friston, K. J., \& Evans, A. C. (1995). A unified statistical approach for determining significant signals in images of cerebral activation. Human Brain Mapping, 4, 58-73.

Yarkoni, T. (2009). Big correlations in little studies: Inflated fMRI correlations reflect low statistical power-commentary on Vul et al. (2009). Perspectives on Psychological Science, 4, 294-298. 